\title{
ABO-Incompatible Living Donor Liver Transplantation in Focus of Antibody Rebound
}

\author{
Silke Rummler ${ }^{a} \quad$ Astrid Bauschke ${ }^{b} \quad$ Erik Baerthel $^{\mathrm{b}}$ Heike Juette $^{\mathrm{a}}$ Katrin Maier $^{\mathrm{a}}$ \\ Christina Malessa $^{\mathrm{b}} \quad$ Dagmar Barz $^{\mathrm{a}} \quad$ Utz Settmacher $^{\mathrm{b}}$ \\ anstitute of Transfusion Medicine, University Hospital Jena, Jena, Germany; \\ ${ }^{\mathrm{b}}$ Department of General, Visceral and Vascular Surgery, University Hospital Jena, Jena, Germany
}

\section{Keywords}

Transplantation - ABO incompatibility · Liver . Antibody rebound

\section{Summary}

Background: Living donor liver transplantation (LDLT) is an option to expand the donor organ pool for patients with life-threatening diseases who cannot be supplied with a cadaver organ. Next to the donor risks, complications after ABO-incompatible LDLT (ABOi LDLT) in the recipient are subject to controversial discussion. Improvement in ABOi graft survival rates have been achieved with plasma treatment procedures (PTP) and immunosuppression but antibody-mediated rejection (AMR) and graft loss still occur. Methods: Since 2008, we have prepared 10 patients for ABOi LDLT. Seven of the 10 patients for transplantation had hepatocellular carcinoma (HCC). Results: All patients underwent PTP before and after ABOi LDLT as well as immunosuppression according to the treatment schedule. We did not use antiCD20 monoclonal antibodies in the transplant setting. We transplanted 6 of 10 preconditioned patients. After 3 years, 5 of the 6 transplanted patients were still alive. Conclusion: Even if B-cell depletion with anti-CD 20 treatment in the setting of ABOi LDLT is commonly accepted, our center successfully administered only quadruple drug immunosuppression combined with PTP. Especially patients with HCC had a high titer increment also pre-transplantation and were at high risk for arterial thrombosis and graft loss.

(c) 2016 S. Karger GmbH, Freiburg

Silke Rummler and Astrid Bauschke contributed equally to this paper.

\section{Introduction}

Due to donor organ shortage in Germany, living donor liver transplantation (LDLT) is an option to expand the donor organ pool for patients who cannot be supplied with a cadaver organ in spite of a life-threatening disease in time. Besides the donor risks, complications after ABO-incompatible living donor liver transplantation (ABOi LDLT) in the recipient are controversially discussed: arterial thrombosis, cellular and antibody-mediated rejection, sepsis and graft failure due to biliary complications as well as sepsis and liver necrosis [1-3].

Although the liver seems to be more resistant to hyperacute rejection than the kidney or heart, hyperacute rejection may occur in pre-sensitized recipients and in recipients of $\mathrm{ABO}$-incompatible (ABOi) allografts [4]. Blood group antigens are not only present on the surface of blood cells, on which they were originally described, but also on the surface of the endothelium of vessels and in large bile ducts [5]. Vascular endothelium and the biliary epithelium of hepatic allografts may continue to express donor blood group antigens up to 150 days after transplantation $[6,7]$. Therefore the ABOi graft may be more susceptible to hepatic artery thrombosis and to immunological bile duct injury $[2,8]$.

Improvement in ABOi graft survival rates has been achieved with plasma treatment procedures (PTP) and immunosuppression regimes. Nevertheless, early antibody-mediated rejections (AMR) and graft loss occur. Here preformed anti-A/B antibodies of the recipient are involved. The amount of acceptable anti-A/B is not standardized for ABOi LDLT. As mentioned in the literature, patients with titers $>1: 16$ underwent PTP before transplantation $[9$, 10]. In many cases, unless methods to maintain low anti-A/B titers after transplantation are used, depletion is only transient, and antibody titers rise again the first days after transplantation (posttransplantation rebound) [11]. This leads to rejection in $90 \%$ of all cases [12].

\section{KARGER}

() 2017 S. Karger GmbH, Freiburg

Fax +497614520714
Dr. med. Silke Rummle 
Unlike in Asia, where LDLT and ABOi LDLT are often the only therapeutic option because of religious beliefs, in Western Europe only a few small case series to ABOi LDLT exist $[1,13,14]$. In Asia, the concept of ABOi LDLT is continuously tracked [15].

Since 1995, LDLT has been performed in the Department of General Visceral and Vascular Surgery at the University Hospital of Jena. The first ABOi LDLT took place in 2008. Here, we present our experience with ABOi LDLT in the perioperative setting.

\section{Material and Methods}

\section{Anti-A/B Testing}

Blood group was determined with commercially available antisera according to standard immunohematologic techniques. Anti-A/B titers were specified by direct agglutination at $22{ }^{\circ} \mathrm{C}$ and by indirect anti-human globulin (AHG) tested at $37^{\circ} \mathrm{C}$ using $\mathrm{A} 1, \mathrm{~A} 2$ or B test red blood cells, neutral gel cards and antiIgG gel cards containing rabbit AHG (DiaMed, Cressier, Switzerland). Titers were recorded as inverted value of the highest plasma dilution giving a weak agglutination reaction $(+1)$.

Titers were converted into whole numbers $(1: 1=1,1: 2=2,1: 4=3,1: 8=4$, $1: 16=5,1: 32=6,1: 64=7,1: 128=8,1: 256=9,1: 512=10,1: 1,024=11,1: 2,048$ $=12$ ) to calculate the titer reduction rate (TRR), and titer increment (TI) according to Wilpert et al. [9]. TRR was calculated as follows:

(titer before treatment - titer directly before transplantation) / total number of treatments

The TRR $>1$ reflects the effectiveness of the treatments. Titer recovery between the end of PTP and the beginning of the next treatment is TI. TI $>2$ describes a high rebound. Titers were measured immediately before and after PTP. We attempted to keep the titers (IgG and IgM) as low as possible at the time of transplantation and below 1:8 during the first 2 weeks post ABOi LDLT. We decided to exclude IgM values from this study, as their titer was in every sample below the IgG titer. After ABOi LDLT, anti-A/B has been measured in the first 2 weeks daily in the morning and afterwards weekly or in case of abnormal findings. As soon as anti-A/B increased, the option of PTP has been discussed for every patient individually with the transplantation team.

\section{Removal of Antibodies by PTP and Treatment Protocols}

A continuous blood cell separator (Cobe Spectra ${ }^{\circledR}$, TerumoBCT, Lakewood, CO, USA) was used to separate the blood. For anticoagulation we used citrate (ACD-A), while controlling ionized calcium, or a mix of citrate and heparin or argatroban.

\section{Selective Immunoadsorption}

Selective immunoadsorption (IA), mainly performed in Western Europe, has not been verified with controlled studies in the setting of ABOi LDLT yet. There are no guidelines available.

During IA, at first, plasma is separated from whole blood and then processed in the immunoadsorbent columns (Glycosorb ${ }^{\circledR}$ ABO, Glycorex Transplantation, Lund, Sweden). On the columns, A or B blood group antigens are linked to a sepharose matrix specifically depleting anti-A/B. Other antibodies remain unaffected, as the Glycosorb-column is highly selective for anti-A/B antibodies. No replacement fluid is required. On average, we treated 2.2 times the plasma volume in each procedure.

\section{Therapeutic Plasma Exchange}

Therapeutic plasma exchange (TPE) is a generally accepted nonselective PTP, which eliminates antibodies in patients with solid organ transplants. The American Society of Apheresis guidelines designates the perioperative use of TPE in ABOi LDLT as a category I with $1 \mathrm{C}$ recommendation [16]. No controlled studies of TPE in ABOi LDLT have been published yet
Table 1. Patients overview

\begin{tabular}{ll}
\hline & Patients, years (range) \\
\hline Mean age, years (range) & $53(33-63)$ \\
\cline { 2 - 2 } & $\mathrm{n}$ \\
\cline { 2 - 2 } Treated patients & 10 \\
Female & 5 \\
Male & 5 \\
Cirrhosis & 1 \\
Cirrhosis with HCC & 7 \\
Cirrhosis with cholangiocarcinoma & 1 \\
Giant hemangioma & 1 \\
\hline
\end{tabular}

We performed TPE in urgent cases and in patients with low titers $(\leq 1: 16)$.

The replacement fluid consisted of a 1:1 mix of therapeutic plasma Octaplas ${ }^{\circledR}$ LG (Octapharma GmbH, Langenfeld, Germany), or fresh frozen pathogenreduced apheresis plasma (Institute of Transfusion Medicine Jena, Germany) and $5 \%$ human albumin (Albunorm ${ }^{\circledR}$; Octapharma $\mathrm{GmbH}$ ). We exclusively used $\mathrm{AB}$ plasma. In each procedure, 1.3 plasma volumes were processed.

\section{Immunosuppression}

All patients received quadruple immunosuppression consisting of steroids, calcineurin inhibitors, antimetabolites, and monoclonal antibodies. We did not use intravenous immunoglobulin and anti-CD20. Steroids were scheduled 7 days before transplantation, with $30 \mathrm{mg} /$ day and $500 \mathrm{mg}$ intraoperatively. Afterwards we continued with $1 \mathrm{mg} / \mathrm{day} / \mathrm{kg}$ body weight (BW) and reduced the dose by 5 mg every 2 days until 7,5 mg was reached. Tacrolimus (Prograf ${ }^{\circledR}$; Astellas Pharma, Tokyo, Japan) was started as pre-emptive immunosuppression 3 days before transplantation at $4 \mathrm{mg}$ twice a day to achieve a plasma level of $10 \mu \mathrm{g} / \mathrm{l}$. Mucophenolate mofetil (Cellcept ${ }^{\circledR}$; Roche Pharmaceuticals, Basel, Switzerland) was also started 3 days before transplantation, $1 \mathrm{~g}$ every $12 \mathrm{~h}$. On the 2 nd and on the 4 th postoperative day the interleukin-2 receptor antagonist Basiliximab (Simulect ${ }^{\circledR}$; Novartis Pharmaceuticals, East Hanover, NJ, USA) was administered by i.v. infusion at $20 \mathrm{mg}$. For 10 days after transplantation, Iloprost (Ilomedin ${ }^{\circledR}$; Bayer Vital GmbH, Leverkusen, Germany) was given at $1 \mathrm{ng} / \mathrm{kg}$ BW.

Patients

Since 2008 until 2013, we have prepared 10 patients with different indications for ABOi LDLT (table 1). The highest initial titer was 1:2,048 (anti-A) and the most often diagnosis HCC.

Written, informed consent for ABOi LDLT and PTP was obtained from each patient.

The positive vote of the local ethics commission was obtained with the number 4337-02/15.

\section{Results}

All patients underwent PTP before ABOi LDLT. On average, we performed 4 PTP (range 1-7) prior to ABOi LDLT, to overcome pre-transplantation rebound, and 6 PTP (0-12) after ABOi LDLT to overcome post-transplantation rebound (table 2). We completed all PTP as scheduled. Except mild citrate reaction, no side events or technical problems occurred. Especially after 3 PTP we observed an increased titer rebound, mainly in carcinoma patients.

Patients 1, 3, and 7 presented no clinical side effects before and after transplantation. By contrast, patients 5, 9, and 10 could not receive $A B O i$ LDLT due to their risk profile and high pre trans- 
Table 2. Blood group combination, diagnosis for ABOi LDLT, TRR ( $>1$ is efficient), mean TI ( $>2$ is high) according to PTP and graft functionality

\begin{tabular}{|c|c|c|c|c|c|c|c|c|c|c|}
\hline Patient & $\begin{array}{l}\text { Blood group } \\
\text { donor } \rightarrow \text { recipient }\end{array}$ & Diagnosis & $\begin{array}{l}\text { Anti-A/B } \\
\text { initial }\end{array}$ & $\begin{array}{l}\text { PTP } \\
\text { pre LDLT }\end{array}$ & TRR & TI & $\begin{array}{l}\text { Anti-A/B } \\
\text { at LDLT }\end{array}$ & $\begin{array}{l}\text { LDLTX } \\
\text { performed }\end{array}$ & $\begin{array}{l}\text { PTP } \\
\text { post LDLT }\end{array}$ & $\begin{array}{l}\text { Survival/graft } \\
\text { function } 2015\end{array}$ \\
\hline 1 & $\mathrm{~A} 1 \rightarrow \mathrm{O}$ & cirrhosis & $1: 2048$ & 5 & 1.0 & 0.59 & $1: 32$ & yes & 3 & yes; $>5$ years \\
\hline 2 & $\mathrm{~A} 1 \mathrm{~B} \rightarrow \mathrm{B}$ & $\mathrm{HCC}$ & $1: 16$ & 2 & 2.5 & 2.0 & negative & yes & 12 & yes; $>3$ years \\
\hline 3 & $\mathrm{~A} 1 \mathrm{~B} \rightarrow \mathrm{A} 1$ & giant hemangioma & $1: 8$ & 1 & 2.0 & 1.5 & $1: 2$ & yes & 0 & yes; $>3$ years \\
\hline 4 & $\mathrm{~A} 1 \mathrm{~B} \rightarrow \mathrm{A} 1$ & $\mathrm{HCC}$ & $1: 128$ & 3 & 1.33 & 2.44 & $1: 1$ & yes & 7 & $\begin{array}{l}\text { no; hepatic } \\
\text { necrosis }\end{array}$ \\
\hline 5 & $\mathrm{~A} 1 \rightarrow \mathrm{O}$ & cholangiocarcinoma & $1: 2048$ & 4 & 1.5 & 2.66 & $1: 32^{*}$ & no & & \\
\hline 6 & $\mathrm{~B} \rightarrow \mathrm{Al}$ & $\mathrm{HCC}$ & $1: 512$ & 4 & 0.66 & 2.0 & $1: 128^{*}$ & no & & \\
\hline 7 & $\mathrm{~A} 1 \mathrm{~B} \rightarrow \mathrm{A} 1$ & $\mathrm{HCC}$ & $1: 8$ & 2 & 3.0 & 1.0 & negative & yes & 1 & yes; $>3$ years \\
\hline 8 & $\mathrm{~A} 2 \rightarrow \mathrm{B}$ & HCC & $1: 32$ & 5 & 0.8 & 1.77 & $1: 2$ & yes & 11 & $\begin{array}{l}\text { no; extrahepatic } \\
\text { metastasis }\end{array}$ \\
\hline 9 & $\mathrm{~A} 1 \rightarrow 0$ & HCC & $1: 512$ & 5 & 0.5 & 3.0 & $1: 32^{*}$ & no & & \\
\hline 10 & $\mathrm{~A} 1 \rightarrow 0$ & $\mathrm{HCC}$ & $1: 512$ & 7 & 0.5 & 2.5 & $1: 16^{*}$ & no & & \\
\hline
\end{tabular}

*Preparation to LDLT has been stopped due to rebound.

plantation titer rebound. For 1 patient (no. 6) the donor organ was no longer available. The other 3 patients (no. 2, 4 and 8 ) rebounded strongly after surgery and were subsequently treated with PTP more often (fig. 1-3). These patients were transplanted despite TI $\geq 2$ or TI $>$ TRR and developed severe complications. One of these patients (no. 4) died due to hepatic failure within 1 week after transplantation and another (patient 8) developed a partial portal vein thrombosis, which resolved in the course of PTP spontaneously.

Five of our 6 transplanted patients developed biliary tract complications within 14 days after ABOi LDLT. All patients were only treated endoscopically.

After 3 years, 5 of the 6 transplanted patients were still alive.

With the use of Glycosorb, we were able to remove 2.1 titer steps by treating the 2.2 -fold plasma volume. TPE shows the same results, even with the treatment of 1.3-fold plasma volume.

\section{Discussion}

We report here our experience with ABOi LDLT in a setting with PTP, using a conventional immunosuppressive protocol without administration of anti-CD20 monoclonal antibodies. In Western Europe only small case series for ABOi LDLT exist [14, 17-19].

In our study 5 of 6 transplanted patients are still in hold of their first transplanted organ 3 years after ABOi LDLT. Yet our study's results are clearly limited by the small number of patients we prepared for ABOi LDLT. Still, all patients with HCC presented a high anti-A/B titer and a strong pre-transplantation rebound prior to ABOi LDLT.

ABOi LDLT has always been a controversial issue. The focus of the debate includes severe cell-mediated rejection, AMR, vascular thrombosis, acute liver necrosis, bile duct complications, and sepsis.

Currently elective ABOi LDLT is performed in Asia with excellent results. There, ABOi LDLT patient survival is close to survival rates of ABO compatible (ABOc) LDLT [20-23]. Due to less deceased organ donors, ABOi LDLT on an elective basis with living donor grafts has been well established, particularly in Japan and South Korea $[22,23]$.

One strategy in ABOi LDLT is to reduce antibody titers with PTP like TPE or IA. Using IA, $50-60 \%$ or $1-2$ titer steps of anti$\mathrm{A} / \mathrm{B}$ antibodies have been removed with each plasma volume. We were able to remove 2.1 titer steps by treating the 2.2 -fold plasma volume. TPE shows the same results, even with the treatment of 1.3-fold plasma volume. Asian centers mainly use TPE. In contrast to TPE, IA is highly selective and, as there is no substitution required, holds no transfusion complications. Still, Glycosorb columns are only for single use and cost-intensive. Unfortunately, during one procedure we cannot treat the plasma as often as needed to erase all antibodies from the blood. The amount of plasma we can treat is limited by the amount of citrate we have to administer to each plasma volume because the patient's compromised liver is very limited in metabolizing citrate.

The decision which type, duration, and frequency of PTP we have used has been individualized based on the initial titers, diagnosis, rebound, and response.

The HCC patients showed high initial titer and rebounded strongly ( $\mathrm{TI} \geq 2$ ) despite immunosuppressant therapy (table 2). Investigations of Peter and Werny [30] indicate distinctly higher values of anti-A/B in patients with severe consuming and tumor diseases than in healthy blood donors. One hypothesis explaining the rebound is that the expression of blood group antigens on the biliary tree alters in pathological conditions [24]. The neoexpression or aberrant expression of A or B substances in malignant cells can boost the production of antibodies [25]. Therefore, the tumor bulk might define the antibody rebound. A big tumor mass could define on one hand a high initial antibody titer and on the other a strong rebound $(\mathrm{TRR}<\mathrm{TI})$. Due to their high pre-transplantation rebound, patient 5, 9 and 10 did not receive ABOi LDLT. Still patient 2, 4 and 8 were transplanted despite their rebound (fig. 1-3). Patient 4 and 8 developed severe complications after ABOi LDLT. 


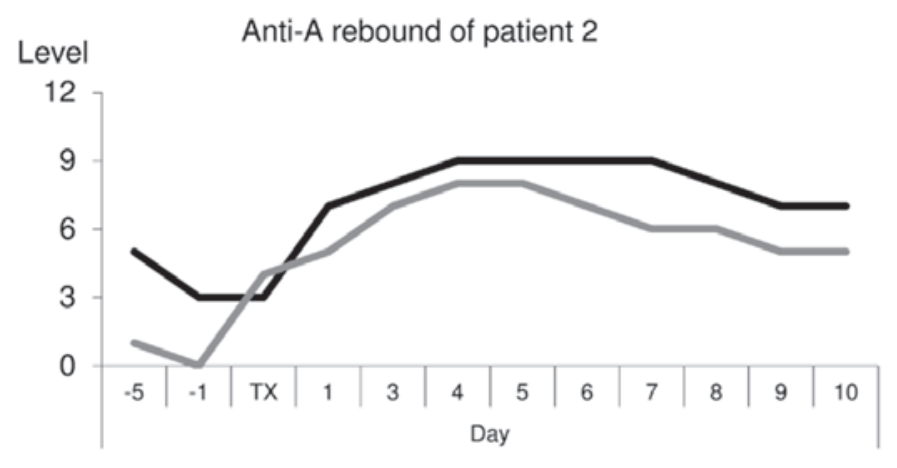

- Anti-A before PTP

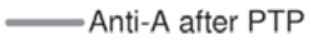

Fig. 1. Anti-A titers pre- and post-PTP are displayed. At transplantation the anti-A titer surprisingly increased significantly.

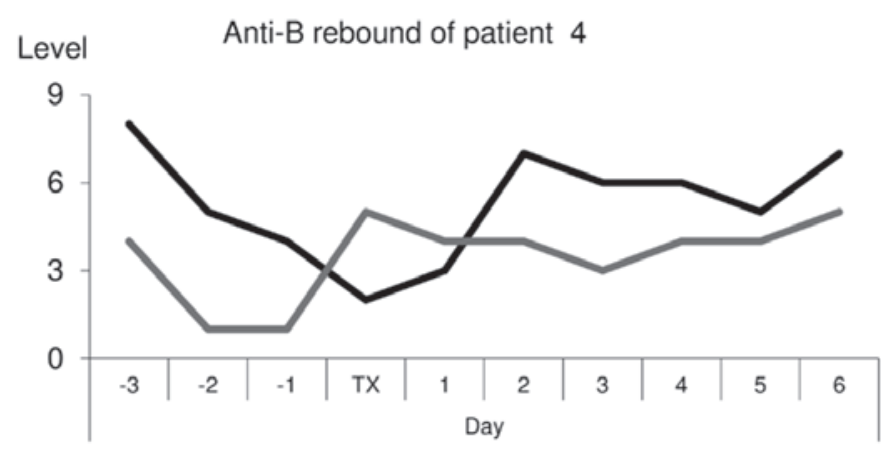

_ Anti-B titre before PTP

Anti-B titre after PTP

Fig. 2. Anti-B titers pre- and post-PTP are displayed. Between the days -1 and +1 antibodies rebounded with TI $>$ TRR.

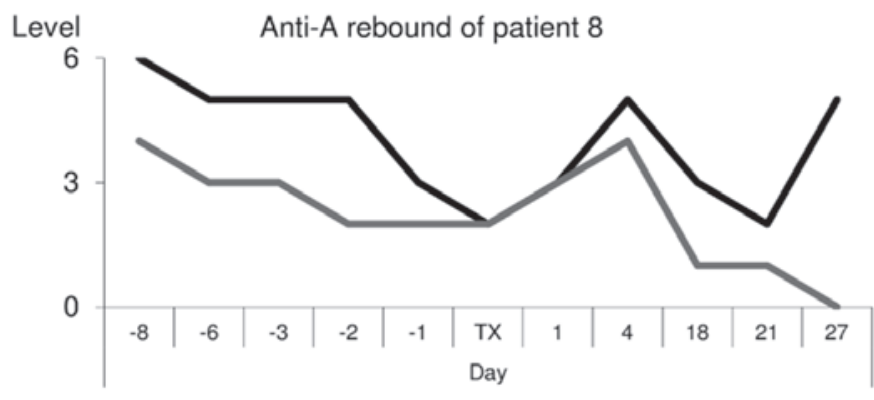

\section{- Anti-B before PTP $\longrightarrow$ Anti-B after PTP}

Fig. 3. Anti-A titers pre- and post-PTP are displayed. On day +1 after LDLT, TRR is equivalent to TI.

Another reason for high initial antibody titers will be immunization against $\mathrm{A}$ and $\mathrm{B}$ substances. This immunization hypothesis is supported by pediatric ABOi liver transplant recipients < 1-yearold having a higher post-transplant survival compared to recipients older than 16 years ( $76 \%$ vs. $22 \%$ ) [26].

In our study anti-A/B rebound occurred mainly after 3 PTP or within 1 week after the first treatment. Additional PTP were necessary to overcome this rebound and achieve the required titer of 1:8 for the surgery day. Prior to ABOi LDLT, we performed on average 4 (1-7) PTP to achieve the target value in $50 \%$ of all cases.

Based on our present experience, currently we consider cancelling further scheduling for transplantation if TRR is below TI in HCC patients after 5 PTP. These patients are at high risk for arterial thrombosis and graft loss. Other transplant centers performed up to 12 PTP before transplantation. No upper limit of PTP has been reported so far [27].

Further, the necessity for splenectomy/anti-CD20 treatment to prevent post-transplantation antibody rebound is still debated. In contrast to Asian protocols, we performed all ABOi LDLTs without splenectomy and without anti-CD20 treatment. Anti-CD20 effectively eliminates CD20-positive B cells up to 6 months, but does not directly affect antibody-producing plasma cells. B cells residing in the lymph nodes stay unaffected by anti-CD20 and are activated by the ABOi graft. As a result, antibody production at low levels is still possible. De novo production of antibodies is sufficiently delayed due to anti-CD20 treatment [28-31]. We assume that splenectomy or anti-CD20 treatment combined with PTP and reinforced immunosuppression can lead to over-immunosuppression and severe systemic infections $[1,28]$. In the study by Thorsen et al. [17], 44 of 66 patients deceased after ABOi LDLT despite antiCD20 administration. Most Asian centers use protocols with antiCD20, plasmapheresis, intravenous immunoglobulin (IVIG), local graft perfusion, and splenectomy [23]. But some Asian centers no longer perform splenectomy routinely $[1,8,15]$. Reports have shown that splenectomy does not offer any immunological benefit in ABOi LDLT. For example Raut et al. [32] observed no statistically significant differences in anti-A/B IgM as well as IgG antibody titers between the 'splenectomy' and the 'non-splenectomy' group. The clinical outcome, including AMR, biliary complications, infections and survival, was also similar in both groups.

Due to the risk of over-immunosuppression, lack of significant benefit and perioperative risks like pancreatic fistula, perioperative morbidity or sepsis, we see no indication for splenectomy. However, IVIG preparations have potent immunoregulatory qualities, too [33]. Urbani et al. [34] described one case of successful use of high-dose IVIG application in ABOi LDLT with AMR. Unfortunately, we observed a transient increase of anti-A/B titers following administration of IVIG and decided to cancel the administration of IVIG in ABOi LDLT treatment schedule. The European Pharmacopoeia recommends anti-A and anti-B to be undetectable in IVIG preparations at 1:64 dilutions [35]. The amount of anti-A/B varies in different preparations. Our own investigations show a variation from 1:1,024 to $1: 16$ for anti-A1 titer, from 1:256 to 1:4 for anti-A2, and from 1:256 to $1: 8$ for anti-B. IVIG infusion leads to a passive transfer of anti-A/B.

PTP are the only effective method to decrease antibody titers after ABOi LDLT [36]. In two cases we were able to save patient and graft with 11 or 12 PTP. Despite reinforced PTP twice a day, patient 4 suffered from hepatic necrosis and died.

Sanchez -Urdazpal et al. [6] reported in 1993 that biliary complication and rejection in deceased patients with ABOi LDLT was much higher than in deceased patients with ABOc LT. Biliary com- 
plications were developed by $54-82 \%$ of the ABOi allograft recipients compared to $6 \%$ in $\mathrm{ABO}$ matched allografts. Hepatic artery thrombosis occurred in $24 \%$ of ABOi allografts [3, 28]. The metaanalysis of Wu et al. [2] in 2011 showed the total complication incidence and acute rejection incidence to be higher after ABOi LDLT than after $\mathrm{ABOc}$ LT. Lee at al. [23] performed ABOi LDLT with a protocol of plasmapheresis, IVIG, and quadruple immunosuppressive therapy (basiliximab, tacrolimus, mycophenolate mofetil, steroids). The authors reported that $5.6 \%$ of the patients developed complications like diffuse intrahepatic biliary strictures (DIHBS) which occurred 2.1-5.2 months post-transplant. The overall biliary complications may be related to direct immunological mechanisms such as bile duct epithelium expressing A and B blood group antigens $[6,7]$. Song et al. [15] also reported a higher incidence of biliary strictures in ABOi LDLT due to DIHBS than in ABOc transplantations. These strictures affected the overall survival significantly.

Living donation provides a curative treatment option for patients with HCC cirrhosis, particularly if a patient is not offered a donor organ by Eurotransplant. This can be due to low labMELD or a tumor burden above the Milan criteria, even if it has benign tumor biology. There are only a few reports on successful ABOi LDLT for patients with HCCs outside Milan criteria [37]. Because of a high early recurrence rate of $57 \%$ in the first year after ABOi LDLT, Lee et al. [38] recommended not to treat HCC patients with
ABOi LDLT. In our series, we transplanted 4 HCC patients (1 outside, 3 inside Milan criteria). The 'outside' patient developed pulmonary metastases within 1 year after ABOi LDLT.

\section{Conclusion}

Anti-CD20 treatment is a known option and possibly influences antibody rebound after transplantation. ABOi LDLT without antiCD20 administration is possible as well, if the primary disease's escalation or severe side effects are feared. In our facility, we have combined PTP and quadruple drug immunosuppression. However, 5 of 6 patients are still in hold of their first transplanted organ after 3 years.

In this study, patients with HCC and a TRR below TI were at high risk for arterial thrombosis and graft loss. These patients should be cancelled for further scheduling.

It remains questionable how transplant risks for HCC patients are to be lowered and how immunosuppression and PTP can be adjusted to overcome the strong rebound prior to transplantation.

\section{Disclosure Statement}

The authors declare no conflict of interest.

\section{References}

1 Zhou J, Ju W, Yuan X, Jiao X, Zhu X, Wang D, He X: ABO-incompatible liver transplantation for severe hepatitis B patients. Transpl Int 2015;28:793-799.

2 Wu J, Ye S, Xu X, Xie H, Zhou L, Zheng S: Recipient outcomes after $\mathrm{ABO}$-incompatible liver transplantation: a systematic review and meta-analysis. PloS One 2011;6:e16521.

3 Detry O: Should ABO-incompatible deceased liver transplantation be reconsidered? Transpl Int 2015;28: 788-789.

4 Hanto DW, Fecteau AH, Alonso MH, Valente JF, Whiting JF: ABO-incompatible liver transplantation with no immunological graft losses using total plasma exchange, splenectomy, and quadruple immunosuppression: evidence for accommodation. Liver Transpl 2003;9:22-30.

5 Ravn V, Dabelsteen E: Tissue distribution of histoblood group antigens. APMIS 2000;108:1-28.

6 Sanchez-Urdazpal L, Batts KP, Gores GJ, Moore SB, Sterioff S, Wiesner RH, Krom RA: Increased bile duct complications in liver transplantation across the ABO barrier. Ann Surg 1993;218:152-158.

7 Nishida S, Nakamura N, Kadono J, Komokata T, Sakata R, Madariaga JR, Tzakis AG: Intrahepatic biliary strictures after liver transplantationJ Hepatobiliary Pancreat Surg 2006;13:511-516.

8 Lee SD, Kim SH, Kong SY, Kim YK, Lee SA, Park SJ: $\mathrm{ABO}$-incompatible living donor liver transplantation without graft local infusion and splenectomy. HPB (Oxford) 2014;16:807-813.
9 Mendes M, Ferreira AC, Ferreira A, Remedio F, Aires I, Cordeiro A, Mascarenhas A, Martins A, Pereira P, Gloria H, Perdigoto R, Veloso J, Ferreira P, Oliveira J, Silva M, Barroso E, Nolasco F: ABO-incompatible liver transplantation in acute liver failure: a single Portuguese center study. Transplant Proc 2013;45:1110-1115.

10 Crew RJ, Ratner LE: ABO-incompatible kidney transplantation: current practice and the decade ahead. Curr Opin Organ Transplant 2010;15:526-530.

11 Reding R, Squifflet JP, Latinne D, De Bruyere M, Pirson Y, Alexandre GP: Early postoperative monitoring of natural anti-A and anti-B isoantibodies in ABO-incompatible living donor renal allografts. Transplant Proc 1987; 19:1989-1990.

12 Colvin RB, Smith RN: Antibody-mediated organ-allograft rejection. Nat Rev Immunol 2005;5:807-817.

13 Umemura A, Nitta H, Sasaki A, Takahara T, Hasegawa Y, Wakabayashi G: ABO-incompatible living donor liver transplantation from hepatitis B core antibody positive donor to hepatitis $\mathrm{C}$ liver cirrhosis recipient: a case report. Case Rep Transplant 2014;2014:507621

14 Goralczyk AD, Obed A, Schnitzbauer A, Doenecke A, Tsui TY, Scherer MN, Ramadori G, Lorf T: Adult living donor liver transplantation with $\mathrm{ABO}$-incompatible grafts: a German single center experience. J Transplant 2009;2009:759581.

15 Song GW, Lee SG, Hwang S, Ahn CS, Moon DB, Kim KH, Ha TY, Jung DH, Park GC, Namgung JM, Park CS, Park HW, Park YH: Successful experiences of ABO-incompatible adult living donor liver transplantation in a single institute: no immunological failure in 10 consecutive cases. Transplant Proc 2013;45:272275.
16 Schwartz J, Winters JL, Padmanabhan A, Balogun RA Delaney M, Linenberger ML, Szczepiorkowski ZM, Williams ME, Wu Y, Shaz BH: Guidelines on the use of therapeutic apheresis in clinical practice-evidencebased approach from the Writing Committee of the American Society for Apheresis: the sixth special issue. J Clin Apher 2013;28:145-284.

17 Thorsen T, Dahlgren US, Aandahl EM, Grzyb K, Karlsen TH, Boberg KM, Rydberg L, Naper C, Foss A Bennet W: Liver transplantation with deceased $\mathrm{ABO}$ incompatible donors is life-saving but associated with increased risk of rejection and post-transplant complications. Transpl International 2015;28:800-812.

18 Schumann A, Fiedler M, Beckebaum S, Cicinnati VR Herzer K, Lenz V, Witzke O, Paul A, Roggendorf M, Horn PA, Lindemann M: Donor- and recipient-derived immunity in $\mathrm{ABO}$ incompatible living-related liver transplantation. Hum Immunol 2015;76:631-635.

19 Broelsch CE, Malago M, Testa G, Valentin Gamazo C Living donor liver transplantation in adults: outcome in Europe. Liver Transpl 2000;6:S64-65.

20 Kim JM, Kwon CH, Joh JW, Kang ES, Park JB, Lee JH Kim SJ, Paik SW, Lee SK, Kim DW: ABO-incompatible living donor liver transplantation is suitable in patients without ABO-matched donor. J Hepatol 2013;59: 1215-1222.

21 Tanabe M, Kawachi S, Obara H, Shinoda M, Hibi T, Kitagawa Y, Wakabayashi G, Shimazu M, Kitajima M Current progress in $\mathrm{ABO}$-incompatible liver transplantation. Eur J Clin Invest 2010;40:943-949.

22 Egawa H, Tanabe K, Fukushima N, Date H, Sugitani A Haga $\mathrm{H}$ : Current status of organ transplantation in Japan. Am J Transplant 2012;12:523-530. 
23 Lee SG: A complete treatment of adult living donor liver transplantation: a review of surgical technique and current challenges to expand indication of patients. Am J Transplant 2015;15:17-38.

24 Nakanuma Y, Sasaki M: Expression of blood grouprelated antigens in the intrahepatic biliary tree and hepatocytes in normal livers and various hepatobiliary diseases. Hepatology 1989;10:174-178.

25 Nydegger UE, Tevaearai H, Berdat P, Rieben R, Carre T, Mohacsi P, Flegel WA: Histo-blood group antigens as allo- and autoantigens. Ann N Y Acad Sci 2005; 1050:40-51.

26 Warner PR, Nester TA: ABO-incompatible solid-organ transplantation. Am J Clin Pathol 2006;125(suppl): S87-94.

27 Wilpert J, Geyer M, Teschner S, Schaefer T, Pisarski P, Schulz-Huotari C, Gropp A, Wisniewski U, Goebel H, Gerke P, Walz G, Donauer J: ABO-incompatible kidney transplantation-proposal of an intensified apheresis strategy for patients with high initial isoagglutinine titers. J Clin Apher 2007;22:314-322.

28 Egawa H, Teramukai S, Haga H, Tanabe M, Mori A, Ikegami T, Kawagishi N, Ohdan H, Kasahara M, Umeshita K: Impact of rituximab desensitization on blood-type-incompatible adult living donor liver transplantation: aJ multicenter study. Am J Transplant 2014;14:102-114.
29 Uchiyama H, Mano Y, Taketomi A, Soejima Y, Yoshizumi T, Ikegami T, Shirabe K, Maehara Y: Kinetics of anti-blood type isoagglutinin titers and B lymphocytes in ABO-incompatible living donor liver transplantation with rituximab and plasma exchange. Transplantation 2011;92:1134-1139.

30 Tyden G, Donauer J, Wadstrom J, Kumlien G, Wilpert J, Nilsson T, Genberg H, Pisarski P, Tufveson G: Implementation of a protocol for $\mathrm{ABO}$-incompatible kidney transplantation - a three-center experience with 60 consecutive transplantations. Transplantation 2007; 83:1153-1155.

31 Schukfeh N, Lenz V, Metzelder ML, Paul A, Mathe Z, Kathemann S, Hoyer PF, Dohna-Schwake C, Gerner P: First case studies of successful ABO-incompatible living-related liver transplantation in infants in Germany. Eur J Pediatr Surg 2015;25:77-81.

32 Raut V, Mori A, Kaido T, Ogura Y, Taku I, Nagai K, Sasaki N, Endo K, Hata T, Yagi S, Egawa H, Uemoto S: Splenectomy does not offer immunological benefits in ABO-incompatible liver transplantation with a preoperative rituximab. Transplantation 2012;93:99-105.

33 Sewell WA, Jolles S: Immunomodulatory action of intravenous immunoglobulin. Immunology 2002;107: 387-393.
34 Urbani L, Mazzoni A, De Simone P, Catalano G, Coletti L, Montin U, Morelli L, Campani D, Pollina L, Biancofiore G, Bindi L, Scatena F, Filipponi F: Treatment of antibody-mediated rejection with high-dose immunoglobulins in $\mathrm{ABO}$-incompatible liver transplant recipient. Transpl Int 2007;20:467-470.

35 Padmore RF: Hemolysis upon intravenous immunoglobulin transfusion. Transfus Apher Sci 2012;46: 93-96.

36 Kozaki K, Egawa H, Kasahara M, Oike F, Yoshizawa A Fukatsu A, Tanaka K: Therapeutic strategy and the role of apheresis therapy for $\mathrm{ABO}$ incompatible living donor liver transplantation. Ther Apher Dial 2005;9: 285-291.

37 Nakamura Y, Hama K, Iwamoto H, Yokoyama T, Kihara Y, Konno O, Jojima Y, Shimazu M: Long-term recurrence-free survival after liver transplantation from an $\mathrm{ABO}$-incompatible living donor for treatment of hepatocellular carcinoma exceeding Milano criteria in a patient with hepatitis B virus cirrhosis: a case report. Transplant Proc 2012;44:565-569.

38 Lee SD, Kim SH, Kong SY, Kim YK, Park SJ: Kinetics of $\mathrm{B}, \mathrm{T}, \mathrm{NK}$ lymphocytes and isoagglutinin titers in $\mathrm{ABO}$ incompatible living donor liver transplantation using rituximab and basiliximab. Transplant Immunol 2015;32:29-34. 